\title{
Stenotrophomonas maltophilia endogenous endophthalmitis: clinical presentation, antibiotic susceptibility, and outcomes
}

\author{
This article was published in the following Dove Press journal: \\ Clinical Ophthalmology \\ 18 August 2014 \\ Number of times this article has been viewed
}

\author{
Jay Chhablani' \\ Aditya Sudhalkar' \\ Animesh Jindal ${ }^{2}$ \\ Taraprasad Das' \\ Swapna R Motukupally ${ }^{3}$ \\ Savitri Sharma ${ }^{3}$ \\ Avinash Pathengay ${ }^{2}$ \\ Harry W Flynn Jr \\ 'Srimati Kannuri Santhamma Centre \\ for Vitreoretinal Diseases, LV \\ Prasad Eye Institute, Kallam Anji \\ Reddy Campus, Hyderabad, India; \\ ${ }^{2} \mathrm{~L} V$ Prasad Eye Institute, GMR \\ Varalakshmi Campus, Visakhapatnam, \\ India; ${ }^{3}$ haveri Microbiology Centre, \\ LV Prasad Eye Institute, Kallam \\ Anji Reddy Campus, Hyderabad, \\ India; ${ }^{4}$ Bascom Palmer Eye Institute, \\ Department of Ophthalmology, \\ University of Miami, Miller School \\ of Medicine, Miami, FL, USA
}

Objective: To describe clinical presentation, antibiotic susceptibility, and outcomes in patients with Stenotrophomonas maltophilia endogenous endophthalmitis.

Design: Retrospective case series.

Participants: Four eyes of four patients with S. maltophilia endogenous endophthalmitis.

Methods: Retrospective chart review of culture-positive S. maltophilia endogenous endophthalmitis treated at L V Prasad Eye Institute, Hyderabad, India, between January 2007 and December 2012, was done. Collected information included demographic, clinical, and microbiology data.

Results: These four patients with S. maltophilia endogenous endophthalmitis cases accounted for $0.47 \%$ (4/836) of total bacterial endophthalmitis cases treated in this period. All patients were from a rural setting and younger than 40 years. Two of the four patients had a history of immune compromise or hospitalization. The visual acuity at presentation was less than 20/320 in all patients. Common presenting features were severe anterior and posterior segment inflammation and hypopyon. All patients underwent vitrectomy with injection of intravitreal antibiotics and dexamethasone. Direct microscopy of the vitreous sample was positive in all cases. All isolates were sensitive to fluoroquinolones and chloramphenicol; sensitivity to aminoglycosides and third-generation cephalosporins was highly variable. The final visual acuity was 20/80 or more in three patients. The time to presentation did not seem to influence the visual or anatomical outcome.

Conclusion: S. maltophilia is a rare cause of endogenous endophthalmitis and usually occurs in young and apparently healthy individuals. Clinical presentation is moderate to severe, and recovery is variable. Fourth-generation fluoroquinolones and chloramphenicol were the most sensitive antibiotics against $S$. maltophilia in this series of patients.

Keywords: Stenotrophomonas maltophilia, endogenous endophthalmitis, Pseudomonas maltophilia, Xanthomonas maltophilia

\section{Introduction}

Stenotrophomonas maltophilia (earlier known as Pseudomonas maltophilia and Xanthomonas maltophilia) is an aerobic, motile, nonfermenting, gram-negative rod, a free-living organism isolated from soil and water that is able to live within amoebae. Because of its ability to colonize on plastic, glass, and Teflon, S. maltophilia has gained significance as a hospital-acquired pathogen. ${ }^{1}$ There are several reports of $S$. maltophilia infection both in ophthalmic and nonophthalmic literature. ${ }^{2,3}$ Ophthalmic infections include conjunctivitis, keratitis, dacryocystitis, and postoperative and traumatic endophthalmitis. ${ }^{4,5}$ We reported one case of $S$. maltophilia endogenous endophthalmitis earlier. ${ }^{6}$ The current report adds an additional four patients from the
Correspondence: Avinash Pathengay

L V Prasad Eye Institute, GMR Varalakshm

Campus, Visakhapatnam, India

$\mathrm{Tel}+9189139892020$

Fax +918913984444

Email avinash@lvpei.org 
same center spread over the course of 5 years (2007-2012). Considering the rarity of such infection and the fact that this organism expresses high levels of intrinsic resistance, we believe the current report will improve management strategies of S. maltophilia infection. .,5,7-11 $^{4}$

\section{Participants and methods}

A retrospective database search was made of medical and laboratory records of L V Prasad Eye Institute, Hyderabad, India, from January 1, 2007, through December 31, 2012. The study was approved by the institutional review board. Search strategies included S. maltophilia and the older terminology and taxonomic nomenclature systems for S. maltophilia (Alcaligenes faecalis, Pseudomonas alcaligenes, Pseudomonas maltophilia, and Xanthomonas maltophilia) to ensure complete data retrieval and analysis.

Data included demographic information, ocular and medical history, ocular and systemic examination, special investigations (if any), time to diagnosis of endophthalmitis, microbiology (direct microscopy and culture), antibiotic susceptibility and resistance patterns of the organism, treatment details, and final outcomes. Undiluted vitreous samples for microbiology were obtained with a vitreous cutter from all patients, as per the standard institute protocol. The microbiological investigation included direct microscopy (Gram, Giemsa, and $\mathrm{KOH}$ with Calcoflour white) and culture (5\% sheep blood agar, 5\% sheep blood chocolate agar, brain heart infusion broth, thioglycollate broth, Sabouraud's dextrose agar, and potato dextrose agar. Blood and urine cultures were also done in all patients. All media were incubated at $37^{\circ} \mathrm{C}$ for 1 week with the exception of Sabouraud's dextrose agar and potato dextrose agar, which were incubated at $27^{\circ} \mathrm{C}$ for 2 weeks. Sheep blood chocolate agar was kept in $5 \% \mathrm{CO}_{2}$ for 1 week. Isolates were identified by conventional biochemical methods and confirmed by Mini API ID 32 GN strips (bioMérieux, Craponne, France; one case) and the Vitek II compact identification system (bioMérieux; three cases). Antibiotic susceptibility testing was performed using the Kirby Bauer disc diffusion method, as per Clinical and Laboratory Standards Institute guidelines.

All patients were managed in consultation with an internist to rule out concurrent systemic foci of infection. After vitreous biopsy, all patients underwent vitrectomy and intravitreal antibiotics injection. Initial intravitreal antibiotics included vancomycin and amikacin or ceftazidime, as per surgeon preference. Repeat intravitreal antibiotics were based on culture sensitivity. Additional procedures, such as retinal detachment surgery, were performed as required on the basis of the individual merits of each case.

\section{Results}

The mean age was $29.7 \pm 4.25$ years (range, $12-35$ years). There were two female and two male patients. Patient 1 had a history of hospitalization, and patient 4 was under long-term treatment with corticosteroids for sarcoidosis. There was no antecedent history, such as trauma, fever, use of intravenous fluids, or urinary tract infection. The mean time to presentation was $10.5 \pm 6.42$ days (range, 6-30 days). The visual acuity at presentation was light perception in two patients: one patient had hand motions and one patient had presenting visual acuity of 20/320. The ocular signs and symptoms of endophthalmitis included lid edema (all four patients), keratic precipitates (two of four patients), hypopyon (all patients; mean height, $0.75 \pm 0.42 \mathrm{~mm}$ ), vitreous exudates (all patients), and an obscured fundus view (all four patients). Cornea and lens were clear at presentation in all patients. An ultrasound B scan examination recorded numerous low- to moderate-intensity echoes throughout the vitreous cavity and significant choroidal thickening, but no retinal detachment at presentation. All patients underwent surgery within 12 hours of presentation, which consisted of pars plana vitrectomy and intravitreal antibiotics (vancomycin and amikacin or ceftazidime). All patients received a second injection of the appropriate antibiotic (vancomycin in three cases and chloramphenicol in one case), and three of four patients received dexamethasone during the second injection.

The sensitivity pattern of $S$. maltophilia strains in this series (Table 1) showed resistance to ceftazidime in two of four instances and to amikacin in one of four instances, as well as to vancomycin in one instance. All strains were sensitive to ciprofloxacin, chloramphenicol, gatifloxacin, and moxifloxacin.

The duration of follow-up, anatomical outcomes, and final visual acuity (at last follow-up) is shown in Table 1. The final vision was hand motions in one patient, and three patients regained 20/80 or better. The final visual acuity was independent of the time to presentation. Patient number 1 developed a retinal detachment approximately a month after surgery but refused further surgical intervention. The fellow eye was normal in all patients.

\section{Discussion}

Endogenous endophthalmitis is less common than postoperative and post-traumatic endophthalmitis. The diagnosis of endogenous endophthalmitis is made when there is no 


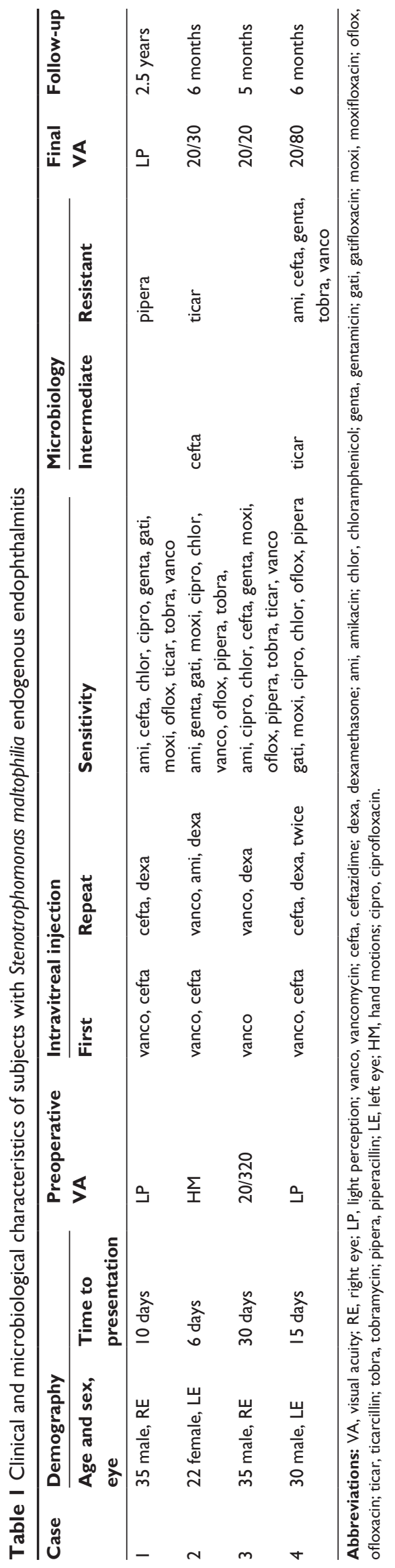

history of any past ocular surgery or trauma in the eye with endophthalmitis. Gram-negative bacterial infection, particularly Klebsiella pneumoniae, as the principal cause of endogenous endophthalmitis is reported from Southeast Asia. ${ }^{12}$ S. maltophilia endophthalmitis after cataract surgery, ${ }^{13,14}$ after ganciclovir implant, ${ }^{8}$ and after ocular injury ${ }^{15}$ has been reported earlier, although $S$. maltophilia as the cause of endogenous endophthalmitis has not been often reported. A report from this institute was the first such case, ${ }^{6}$ and is currently followed with four additional cases of endogenous endophthalmitis caused by $S$. maltophilia.

The absence of ocular or systemic compromise in two patients in this series is of particular interest. Blood and urine cultures were negative, and history was not suggestive of a systemic source of infection.

Antibiotic sensitivity of S. maltophilia is variable. ${ }^{48,10,11,13,15-17}$ Chang et $\mathrm{l}^{13}$ have recently published clinical and microbiological results of eight cases of S. maltophilia endophthalmitis after cataract surgery. In their series, seven of seven isolates tested were susceptible to ceftazidime. Two of seven cases tested were resistant to ciprofloxacin. In our first case report, ${ }^{6}$ S. maltophilia was intermediate sensitive or resistant to ceftazidime, which is currently recommended for empiric coverage of gram-negative infection. In this series, it also was resistant to ceftazidime in three cases and to amikacin in one case. It was sensitive to chloramphenicol, ciprofloxacin, and gatifloxacin in this series and in an earlier reported case. On the basis of our report, we suggest intravitreal chloramphenicol and oral ciprofloxacin in the management of S. maltophilia causing endogenous endophthalmitis. The safety of intravitreal chloramphenicol has been reported in the literature. ${ }^{18}$ Use of intravitreal dexamethasone that is known to reduce inflammation can also be useful. ${ }^{9}$

To conclude, S. maltophilia endogenous endophthalmitis can occur in young and apparently healthy individuals. Clinical presentation of S. maltophilia endogenous endophthalmitis is moderate to severe in nature, in line with other gram-negative organisms. Intravitreal chloramphenicol may be the drug of choice in this infection.

\section{Author contributions}

JC and AS carried out the data collection and data analysis. JC, $\mathrm{AS}$, and $\mathrm{AJ}$ drafted the manuscript. AP is one of the treating physicians and also carried out the correction of the manuscript. TD is the other treating physician. SRM and SS are the microbiologists. HWF corrected the manuscript. All authors contributed toward data analysis, drafting and revising the paper and agree to be accountable for all aspects of the work. 


\section{Disclosure}

The authors report no conflicts of interest in this work.

\section{References}

1. Jucker BA, Harms H, Zehnder AJ. Adhesion of the positively charged bacterium Stenotrophomonas (Xanthomonas) maltophilia 70401 to glass and Teflon. J Bacteriol. 1996;178(18):5472-5479.

2. Ballestero S, Vírseda I, Escobar H, Suárez L, Baquero F. Stenotrophomonas maltophilia in cystic fibrosis patients. Eur J Clin Microbiol Infect Dis. 1995;14(8):728-729.

3. Baltimore RS, Jenson HB. Puncture wound osteochondritis of the foot caused by Pseudomonas maltophilia. Pediatr Infect Dis J. 1990;9(2): 143-144.

4. Denton M, Kerr KG. Microbiological and clinical aspects of infection associated with Stenotrophomonas maltophilia. Clin Microbiol Rev. 1998;11(1):57-80.

5. Penland RL, Wilhelmus KR. Stenotrophomonas maltophilia ocular infections. Arch Ophthalmol. 1996;114(4):433-436.

6. Das T, Deshmukh HS, Mathai A, Reddy AK. Stenotrophomonas maltophilia endogenous endophthalmitis: clinical presentation, sensitivity spectrum and management. J Med Microbiol. 2009;58(Pt 6):837-838.

7. Chen KJ, Wang NK, Sun MH, et al. Endophthalmitis caused by Stenotrophomonas maltophilia. Ophthalmic Surg Lasers Imaging. 2010;41(5): e555-e561.

8. Chen S, Stroh EM, Wald K, Jalkh A. Xanthomonas maltophilia endophthalmitis after implantation of sustained-release ganciclovir. Am J Ophthalmol. 1992;114(6):772-773.

9. Das T, Jalali S, Gothwal VK, Sharma S, Naduvilath TJ. Intravitreal dexamethasone in exogenous bacterial endophthalmitis: results of a prospective randomised study. Br J Ophthalmol. 1999;83(9):1050-1055.
10. Jackson TL, Eykyn SJ, Graham EM, Stanford MR. Endogenous bacterial endophthalmitis: a 17-year prospective series and review of 267 reported cases. Surv Ophthalmol. 2003;48(4):403-423.

11. Kaiser GM, Tso PC, Morris R, McCurdy D. Xanthomonas maltophilia endophthalmitis after cataract extraction. Am J Ophthalmol. 1997; 123(3):410-411.

12. Wong JS, Chan TK, Lee HM, Chee SP. Endogenous bacterial endophthalmitis: an east Asian experience and a reappraisal of a severe ocular affliction. Ophthalmology. 2000;107(8):1483-1491.

13. Chang JS, Flynn HW Jr, Miller D, Smiddy WE. Stenotrophomonas maltophilia endophthalmitis following cataract surgery: clinical and microbiological results. Clin Ophthalmol. 2013;7:771-777.

14. Karakurt A, Abdik O, Sengün A, et al. Stenotrophomonas maltophilia Endophthalmitis after cataract extraction. Ocul Immunol Inflamm. 2006;14(1):41-46.

15. Lai TY, Kwok AK, Fung KS, Chan WM, Fan DS, Lam DS. Stenotrophomonas maltophilia endophthalmitis after penetrating injury by a wooden splinter. Eye (Lond). 2001;15(Pt 3):353-354.

16. Horio N, Horiguchi M, Murakami K, Yamamoto E, Miyake Y. Stenotrophomonas maltophilia endophthalmitis after intraocular lens implantation. Graefes Arch Clin Exp Ophthalmol. 2000;238(4):299-301.

17. Horster S, Bader L, Seybold U, Eschler I, Riedel KG, Bogner JR. Stenotrophomonas maltophilia induced post-cataract-surgery endophthalmitis: Outbreak investigation and clinical courses of 26 patients. Infection. 2009;37(2):117-122.

18. Koziol J, Peyman G. Intraocular chloramphenicol and bacterial endophthalmitis. Can J Ophthalmol. 1974;9(3):316-321.
Clinical Ophthalmology

\section{Publish your work in this journal}

Clinical Ophthalmology is an international, peer-reviewed journal covering all subspecialties within ophthalmology. Key topics include: Optometry; Visual science; Pharmacology and drug therapy in eye diseases; Basic Sciences; Primary and Secondary eye care; Patient Safety and Quality of Care Improvements. This journal is indexed on Submit your manuscript here: http://www.dovepress.com/clinical-ophthalmology-journal

\section{Dovepress}

PubMed Central and CAS, and is the official journal of The Society of Clinical Ophthalmology (SCO). The manuscript management system is completely online and includes a very quick and fair peer-review system, which is all easy to use. Visit http://www.dovepress.com/ testimonials.php to read real quotes from published authors. 